OC21

SAFE WATER VERSUS NORMAL SALINE NASOPHYRYNX WASH IN THE PREVENTION OF COMMON COLD AMONG CHILDREN: A STRATIFIED RANDOMISED CONTROL TRIAL

Faheem Khan*. Sindh University, Karachi, Pakistan

10.1136/archdischild-2019-epa.20

Introduction Nasal rinsing with normal saline water is prescribe in paediatric practices in prevention of the upper respiratory infection (URTIs) in Pakistan. Safe water is not worse in effectiveness to normal saline effectiveness in nasophyrnx rinsing in primary prevention of common cold has not been established by trials.

Methods Double blind stratified randomized controlled trial carried out in August 2017-January 2018 winter season in Karachi, Pakistan. 774 healthy children aged 02 months to 5 years were included,randomly assigned to nasal wash with normal saline, safe water group at least 3 times a day followedup 90 days. Features of URTIs recorded on check list. Per protocol analysis.

Findings 260 children contracted URTIs. Incidence rate of URTI was 0.52 episodes per 60 person days among control. Incidence rate decreased to 0.34 episodes per 60 person days in the safe water group and 0.48 episodes per 60 person days in the normal saline group. Non inferiority test value were $0.997(90 \% \mathrm{CI}=0.904-1.101)$. Safe water rinsing tended to decrease nasal symptoms $(\mathrm{p}=0.055)$. As hazard ratio $=0.60$, 95\% CI $=0.39-0.95$

Innovations Safe water rinsing was non inferior in effectiveness to normal saline in prevention of URTIs among healthy children. Safe water is cost effective modality would greater benefit the society.

\section{OC22 PARENTAL RATING OF FOLLOW-UP CARE FOR THEIR VERY PRETERM CHILDREN IN EUROPE}

\footnotetext{
1,2Anna-Veera Seppanen ${ }^{*}$, ${ }^{3}$ Elizabeth Draper, ${ }^{1,2}$ Rym El Rafei, ${ }^{4}$ Marina Cuttini, ${ }^{5}$ Stavros Petrou, ${ }^{6}$ Henrique Barros, ${ }^{1}$ Jennifer Zeitlin. ${ }^{1}$ French National Institute of Health and Medical Research (INSERM U1153), Paris, France; ${ }^{2}$ Sorbonne University, Paris, France; ${ }^{3}$ University of Leicester, Leicester, UK; ${ }^{4}$ Bambino Gesu Children's Hospital, Rome, Italy; ${ }^{5}$ University of Warwick, Coventry, UK; ${ }^{6}$ Institute of Public Health University of Porto (ISPUP), Porto, Portugal
}

\subsection{6/archdischild-2019-epa.21}

Background Infants born very preterm are at risk of developing multiple health and developmental problems. Because the prognosis of each individual child is unknown at discharge, follow-up programs are essential for identifying health needs early, enable timely intervention and coordinate health services from multiple providers. Despite their recognized importance, there have been few evaluations of these programs, in particular among parents, whose involvement is crucial for successful follow-up. This study investigated how parents rate their very preterm children's follow-up care in Europe.

Methods The data come from the Screening to improve Health In very Preterm infantS (SHIPS) study, which followed up the area-based EPICE cohort of infants born before 32 weeks ${ }^{6}$ gestation in 2011/12 in regions in 11 European countries. Perinatal data were abstracted from medical records and socioeconomic and child health data were collected with parent-report questionnaires at 2 and 5 years. At 5 years, parents rated the follow-up care received for their child's prematurity (poor, fair, good, excellent) and provided suggestions for improvement as free-text comments. We measured the percentage of poor or fair ratings and associated factors, including country, maternal sociodemographic characteristics, perinatal characteristics (gestational age, neonatal morbidities) and current health and developmental problems.

Results Questionnaires were filled in for 3414 children $(51 \%$ response rate), by mothers (84\%), fathers (14\%) and other caretakers. 93\% reported receiving follow-up care for their child's prematurity. A low percentage (13.7\%) judged followup care to be poor or fair, but this varied from $<10 \%$ in France and the Netherlands to $>20 \%$ in Denmark and Poland $(\mathrm{p}<0.001)$. Higher maternal education was related to more dissatisfaction $(\mathrm{p}<0.01)$. Negative appreciations of care were highest when children had diagnosed health problems, especially cerebral palsy (32.2\%) and developmental delay (28.5\%). After adjustment for current diagnoses, perinatal characteristics were not significantly related to care ratings. Common themes highlighted in free-text comments (from 1032 parents) included the need for longer-term follow-up, focusing on more than physical health and lack of knowledge about prematurity among general practitioners. Some themes were mentioned more frequently in some countries, such as waiting times (Poland), lack of care coordination (Sweden) and length of maternity leave (Portugal).

Conclusions Dissatisfaction with follow-up care was low overall, but was higher among those most reliant on health services. Many common themes emerged from parents' comments despite geographic heterogeneity. Further research is needed to understand differences in reported satisfaction between countries and by maternal educational level.

\section{OC23 THE IMPLEMENTATION OF A CROSS-JURISDICTIONAL CLINICAL NETWORK FOR CONGENITAL HEART DISEASE IN CHILDREN AND YOUNG PEOPLE}

Sharon Morrow*. Children's Heath Ireland, Dublin, Ireland. Royal Belfast Hospital for Sick Children, Belfast, UK

\subsection{6/archdischild-2019-epa.22}

Purpose To develop an All-Island Congenital Heart Disease (CHD) Network to provide high quality and timely access to specialist cardiac services for all children on the island of Ireland.

The core objectives of the All-Island CHD Network are:

Appropriate CHD treatment for all children and young people on the island as close to home as appropriate.

Timely access to quality treatment through the creation of a single waitlist

Provision of a safe and sustainable model of care

Development of a research and innovation hub that delivers best practice solutions

Methods In 2014, an International Working Group Report recommended the cessation of surgery in Northern Ireland (NI) for Paediatric CHD, leading to children from NI travelling to Great Britain (GB) to receive emergency, urgent and elective surgical intervention, including cardiac catherization, a significant risk to the child and at great cost to the health service and families. There was Ministerial acceptance of the recommendations, which led to the creation of an All-Island CHD Network to enable CHD services in NI and Republic of Ireland (ROI) collaborate and work as a single Network to 